\title{
No evidence that common genetic risk variation is shared between schizophrenia and autism
}

Running head title: Common variants in schizophrenia and autism

Jacob A.S. Vorstman, ${ }^{1}$ Richard J.L. Anney, ${ }^{2}$ Eske Derks, ${ }^{1,3}$ Louise Gallagher, ${ }^{2}$ Michael Gill, ${ }^{2}$ Maretha V. de Jonge, ${ }^{1}$ Herman van Engeland, ${ }^{1}$ René S. Kahn ${ }^{1}$, Roel A, Ophoff, ${ }^{1,4}$ the Autism Genome Project, the International Schizophrenia Consortium

${ }^{1}$ Rudolf Magnus Institute of Neuroscience, Department of Psychiatry, University Medical Center Utrecht, Utrecht, The Netherlands

2 Department of Psychiatry, Trinity College Dublin, Dublin, Ireland

${ }^{3}$ Academic Medical Center, Department of Psychiatry, Amsterdam, The Netherlands

${ }^{4}$ Center for Neurobehavioral Genetics, University of California Los Angeles, Los Angeles, Califirnia

Corresponding author: J.A.S Vorstman, J.A.S.Vorstman@UMCUtrecht.nl

Rudolf Magnus Institute of Neuroscience, Department of Psychiatry, University Medical Center Utrecht, Utrecht, 3584 CG, the Netherlands

\begin{abstract}
The similarity between aspects of the clinical presentation of schizophrenia and autism spectrum disorders (ASD) suggests that elements of the biological etiology may also be shared between these two disorders. Recently, an increasing number of rare, mostly structural genetic variants are reported to increase the risk of both schizophrenia and autism spectrum disorders. We hypothesized that given this evidence for a shared genetic background based on rare genetic variants, common risk alleles may also be shared between ASD and schizophrenia. To test this hypothesis, the polygenic score, which summarizes the collective effect of a large number of common risk alleles, was used. We examined whether the polygenic score derived from a schizophrenia case-control dataset, previously reported by Purcell et al., was able to differentiate ASD cases from controls. The results demonstrate that the schizophrenia-derived polygenic score is not different between ASD cases and controls, indicating that there is no important sharing of common risk alleles between the two neuropsychiatric disorders. Possibly, common risk alleles are less important in ASD in comparison to their more prominent role in schizophrenia and bipolar disorders. These findings provide important novel insights into shared and distinct elements of the genetic architecture of autism and schizophrenia.
\end{abstract}

\section{Key words}

Autism, Schizophrenia, Polygenic Risk Score, Common Genetic Variants 


\section{Introduction}

Autism spectrum disorder (ASD) is a highly familial disorder with siblings of a proband showing an 20-fold higher prevalence than that of the general population [Ozonoff et al., 2011]. Ever since its first description in 1943 [Kanner, 1968], the question whether autism ought to be considered as related to, or distinct from schizophrenia has been the subject to debate [Crespi et al., 2008; Konstantareas et al., 2001]. While both disorders clearly have distinguishing symptoms (e.g. positive symptoms in schizophrenia, repetitive behaviors in autism), they also both share core deficits, i.e. social impairment [Sasson et al., 2011]. Even so, the overall consensus since the early 1970s is that autism and schizophrenia are to be regarded as separate entities [Rutter, 1972; Rutter, 1968]. More recent observations, however, suggest that there may be more overlap than previously assumed. Some studies have found that children with ASDs are at increased risk to develop schizophrenia later in life [Mouridsen et al., 2008b; Mouridsen et al., 2008a; Stahlberg et al., 2004], although this finding is not consistently replicated [Skokauskas et al., 2010]. Additionally, the rate of schizophrenia has been found to be elevated in family members of autistic probands [Ghaziuddin, 2005; Larsson et al., 2005; Daniels et al., 2008]. Recently, rare structural genetic variation, mostly copy number variants (CNVs) has been shown to be enriched in individuals with autism [Vorstman et al., 2006; Pinto et al., 2010; Sanders et al., 2011] and with schizophrenia [International Schizophrenia Consortium, 2008; Stefansson et al., 2008; Walsh et al., 2008; Buizer-Voskamp et al., 2011]. Importantly, there is increasing evidence that specific CNVs are associated with increased rates of both ASD and schizophrenia [Girirajan et al., 2010; Merikangas et al., 2009].

While the role of these rare alleles with relatively large effect becomes increasingly clear in ASD, the part played by common variants of smaller effect size is less well defined. With regard to common alleles, the findings in schizophrenia appear more promising in comparison to ASD [Devlin et al., 2011; Cichon et al., 2009]. In 2009, findings reported by the International Schizophrenia Consortium (ISC) suggested that there may be thousands of common genetic variants of very small effect which collectively can contribute to the risk of schizophrenia [Purcell et al., 2009]. Interestingly, the common polygenic variation contributing to the risk of schizophrenia was in part shared with bipolar disorder but had no predictive value for a number of physical diseases including Rheumatoid Arthritis, and Type 1 and 2 Diabetes. The model of polygenic risk scores explaining disease susceptibility has now been successfully applied to a number of phenotypes including multiple sclerosis and height [Bush et al., 2010; Yang et al., 2010]. Given the clinical overlap and the evidence for shared rare genomic CNVs between schizophrenia and ASD, we examined whether common risk alleles may also be shared these two disorders. 


\section{Materials and Methods}

The Autism Genome Project (AGP) dataset consists of proband-parent trios; for the current analysis 2,737 probands with Autism Spectrum Disorder (ASD) were included. Methods of diagnostic assessment and DNA collection have been described elsewhere [Anney et al., 2010]. The samples were genotyped using either the Illumina Infinium 1Msingle SNP microarray or the Illumina 1M-duo microarray. All quality control (QC) procedures were maintained across datasets and are described elsewhere [Anney et al., 2010]. In addition, to reduce array discrepancies, all SNP markers showing a fixation index (FsT) greater than 0.02 across Illumina platforms were excluded from the analysis. Written consent was obtained from all families and procedures had approval from institutional review boards.

We used the same schizophrenia score allele set previously described by the ISC [Purcell et al., 2009]. In short, a subset of 74,062 SNPs was defined based on nominal association at different significance thresholds in a discovery GWAS schizophrenia case control sample. For each individual putative risk variant or "score allele" a relative weight was calculated on the basis of the strength of the association to the disease status in the discovery case-control sample. The weight was given as the log odds ratio of the association signal. In subsequent independent case-control samples, individual polygenic scores are equal to the sum of all risk variants carried by that individual, while taking into account the previously defined score allele weights. When the mean polygenic risk score in cases is significantly higher than the mean in controls, the polygenic score can be considered to index risk for the disorder. All polygene scores were calculated using the 'score' routine in PLINK [Purcell et al., 2007]. Given the absence of unrelated controls in the AGP dataset, the non-transmitted alleles to the proband were used as controls (or pseudocontrols). Pseudo-controls were generated from using the '- -tucc' routine in PLINK [Purcell et al., 2007].

To account for strand differences across studies, all SNPs were mapped to consistent nomenclature, chromosome and position using build b130 of dbSNP as reference [Sherry et al., 2001]. In order to assure adequate quality of the data, SNPs where strand ambiguity was possible were excluded from analysis (A/T or $C / G)$. Allele frequencies were compared between ISC and AGP to support correct re-coding. Following QC, and as a consequence of the ISC and AGP studies being performed on different genotyping platforms, 33,603 of the 74,062 markers identified from the ISC Affymetrix-generated polygene score were mapped to AGP Illumina-generated data (see suppl. figure 1). To exclude any influence of the HLA-region on chromosome 6, a supplementary analysis generating scores excluding this region was also performed (33,457 SNPs).

Logistic regression was used to examine the predictive strength of the schizophreniaderived polygenic score in the ASD case-pseudo control sample, calculating based on two models: 
(1) Case status predicted by score, non-missing genotypes, and 4-degrees of population eigenvalues.

(2) Case status predicted by non-missing genotypes and 4-degrees of population eigenvalues.

A pseudo- $\mathrm{R}^{2}$ (Nagelkerke's) was calculated for each model; the Vm (pseudo-variance at the marker) was calculated as the $R^{2}(1)-R^{2}(2)$. As a pseudo-R2 was used (which is sample specific and not comparable across studies); the significance of each of the calculated Vms was estimated using within-family permutation $(n=1000)$.

\section{Results}

We explored the predictive power of the schizophrenia-derived polygenic score, based on the ISC dataset at 10 association thresholds $\left(P_{T}<0.5,0.4,0.3,0.2,0.1,0.05,0.01,0.001\right.$, 0.0001 and 0.00001 ) in 2736 ASD individuals and matched pseudo-controls. The schizophrenia-derived polygene score was not significantly different between ASD-cases and pseudo-controls for any of the thresholds (see Table I). Moreover, the influence of HLA, a well-replicated association locus for schizophrenia, was negligible. Figure 1 shows the results of our analysis jointly with the previously reported findings of the ISC study in which $3 \%$ of the variance in schizophrenia case-control cohorts and almost $2 \%$ for bipolar disorder was explained based on the 74,062 schizophrenia risk alleles (see Figure 1).

\section{Discussion}

Using the model of common polygenic variation contributing to disease risk, we found no evidence for sharing of common genetic risk variants between schizophrenia and ASD. Previous studies have provided clinical evidence for shared etiology, and molecular evidence for the role of specific rare alleles in both neuropsychiatric traits. In contrast, our results suggest distinct genetic mechanisms underlying ASD and schizophrenia in the case of common variants.

One could argue that the paucity of identified common risk alleles in ASD reduces a priori the probability of success of finding a collective effect of SZC common variants on ASD risk, such as examined in the present study. However, it is important to differentiate the identification of significantly associated risk variants using GWAS from the assessment of risk prediction by a polygenic risk score based on the en masse effect of nominally associated SNPS. Similar to the situation in ASD, for the analyses of Purcell and colleagues, for SCZ there were at most only a few identified significantly associated genetic markers discovered through GWAS, nevertheless, the combined measure of all nominally significant SNPs could explain a small but significant proportion of the variance. Therefore, the scarcity of significant GWAS findings in ASD does not rule out the possibility of a relevant polygenic effect on ASD risk or across-disorder effect. 
Our observations however, indicate that common risk variants for schizophrenia do not index the risk for autism. A possible explanation may be that the role of common risk variants in autism in comparison to schizophrenia and bipolar disorder is limited. Indeed, recent work indicates that the variance explained by a polygenic risk score in ASD is substantially smaller than the explained variance reported in schizophrenia [Anney et al., 2012]. This would, in combination with previously reported findings, support a continuum model of neuropsychiatric disease such as proposed by Craddock et al. [Craddock et al., 2010]. In this model, the psychiatric phenotypes are ordered along a gradient of decreasing neurodevelopmental impairment, starting with mental retardation, then through autism, schizophrenia and bipolar disorder, ending with unipolar mood disorders [Craddock \& Owen, 2010]. The data from CNV studies suggest that higher impact rare genetic variants are more likely to lead to the more severe phenotypes, i.e. mental retardation, autism and schizophrenia. Our results support the complementary assertion as well, that the role of common risk variants may be more prominent for the traits at the milder end of the same spectrum (Figure 2). One possible explanation would be that fecundity is most reduced in the more severe disorders, thus preventing genetic risk variants from becoming common in the population.

It is entirely possible that a proportion of autistic children in the AGP collection may go on to develop schizophrenia later in life. Theoretically, this subgroup could be expected to carry more risk alleles that are also included in the schizophrenia polygenic risk score. In turn, one would expect that this group would upwardly bias these variance estimates, thereby explaining more of the variance in the ASD group due to clinical misclassification in these individuals who have yet to develop schizophrenia. However, the lack of discernible variance explained in these analyses may preclude these assumptions and reflect that there is both negligible shared genetic liability based on common variants between the disorders and a negligible proportion of this cohort that shares liability through misclassification in these individuals.

This study has several strengths and limitations: the relatively large study sample can be considered a strength, with the number of subjects exceeding the sample sizes of the original studies on polygenic score in schizophrenia which were sufficiently powered to detect significantly explained variance of less than 1\% [Purcell et al., 2009; Ruderfer et al., 2011]. Consequently, the expectation to detect a similar crossover association in the present study was reasonable, given the sample size. The use of the same schizophreniaderived polygenic score provided by the ISC is also a strength of the current study although the lack of consistency of SNP arrays between studies limited the overlap of markers reported by the ISC polygenic score and the AGP study. However, in the original paper, results were similar when a set of 25,215 SNPs was used instead of 74,062 SNPs [Purcell et al., 2009], indicating that using 33,603 SNPs with complete genotyping, the current study was sufficiently powered to examine the possibility of shared common risk alleles between schizophrenia and ASD. Moreover, using Beagle [Browning et al., 2009], 
we were able to increase the overlap by imputing an additional 25K SNPs at sufficient quality (>0.6). We found no significant increase in signal in these analyses (data not shown). There are no important confounding variables that may have affected our analyses. It has been suggested that cryptic population stratification could potentially exert a major effect on the results obtained with the analysis of common polygenic variation [McClellan et al., 2010]. Our use of a family-based design, constructing pseudo-controls from un-transmitted parental chromosomes circumvents this issue and has been shown previously to be a reliable method [Ruderfer et al., 2011].

In conclusion, we show that the schizophrenia-derived (ISC) polygenic score does not contribute to the risk of ASDs, suggesting that there is no substantial sharing of common risk alleles between schizophrenia and ASDs. There may be common risk alleles for ASDs that do not contribute to schizophrenia susceptibility. Alternatively, the findings reported here may indicate that in comparison to schizophrenia and bipolar disorder, common risk alleles do not play as prominent a role in ASDs. This alternative explanation is both plausible and consistent with the current lack of confirmed GWAS results in ASDs [Devlin et al., 2011].

\section{Acknowledgments}

This work was supported by a 2010 Young Investigator Award from the National Alliance for Research on Schizophrenia and Depression (NARSAD) and by a 2010 Fellowship from the Dutch Brain Foundation (F2010(1)-20) to JASV, the Health Research Board (Ireland) and Autism Speaks (USA) to RJLA, the Netherlands Scientific Organization (NWO; project number 451-08-010) to ED and the main funders of the AGP: Autism Speaks (USA), the Health Research Board (HRB, Ireland; AUT/2006/1, AUT/2006/2, PD/2006/48), The Medical Research Council (MRC, UK), Genome Canada/Ontario Genomics Institute and the Hilibrand Foundation (USA). Analysis and data management support was provided by the Trinity Centre for High Performance Computing (http://www.tchpc.tcd.ie/) funded through grants from Science Foundation Ireland.

\section{References}

Anney R, Klei L, Pinto D, Regan R, Conroy J, Magalhaes TR, Correia C, Abrahams BS, Sykes N, Pagnamenta AT, et al. 2010. A genomewide scan for common alleles affecting risk for autism. Hum Mol Genet 19:4072-4082.

Anney R, Klei L, Pinto D, Almeida J, Bacchelli E, Baird G, Bolshakova N, Bolte S, Bolton PF, Bourgeron $\mathrm{T}$, et al. 2012. Individual common variants exert weak effects on the risk for autism spectrum disorder. Hum Mol Genet 21:4781-4792.

Browning BL, Browning SR. 2009. A unified approach to genotype imputation and haplotype-phase inference for large data sets of trios and unrelated individuals. Am J Hum Genet 84:210-223.

Buizer-Voskamp JE, Muntjewerff JW, Strengman E, Sabatti C, Stefansson H, Vorstman JA, Ophoff RA. 2011. Genome-Wide Analysis Shows Increased Frequency of Copy Number Variation Deletions in Dutch Schizophrenia Patients. Biol Psychiatry 70:655-662.

Bush WS, Sawcer SJ, De Jager PL, Oksenberg JR, McCauley JL, Pericak-Vance MA, Haines JL. 2010. Evidence for polygenic susceptibility to multiple sclerosis--the shape of things to come. Am J Hum Genet 86:621-625. 
Cichon S, Craddock N, Daly M, Faraone SV, Gejman PV, Kelsoe J, Lehner T, Levinson DF, Moran A, Sklar P, et al. 2009. Genomewide association studies: history, rationale, and prospects for psychiatric disorders. Am J Psychiatry 166:540-556.

Craddock N, Owen MJ. 2010. The Kraepelinian dichotomy - going, going... but still not gone. $\mathrm{Br} \mathrm{J}$ Psychiatry 196:92-95.

Crespi B, Badcock C. 2008. Psychosis and autism as diametrical disorders of the social brain. Behav Brain Sci 31:241-261.

Daniels JL, Forssen U, Hultman CM, Cnattingius S, Savitz DA, Feychting M, Sparen P. 2008. Parental psychiatric disorders associated with autism spectrum disorders in the offspring. Pediatrics 121:e1357-e1362.

Devlin B, Melhem N, Roeder K. 2011. Do common variants play a role in risk for autism? Evidence and theoretical musings. Brain Res 1380:78-84.

Ghaziuddin M. 2005. A family history study of Asperger syndrome. J Autism Dev Disord 35:177182.

Girirajan S, Eichler EE. 2010. Phenotypic variability and genetic susceptibility to genomic disorders. Hum Mol Genet 19:R176-R187.

International Schizophrenia Consortium. 2008. Rare chromosomal deletions and duplications increase risk of schizophrenia. Nature 455:237-241.

Kanner L. 1968. Autistic disturbances of affective contact. Acta Paedopsychiatr 35:100-136.

Konstantareas MM, Hewitt T. 2001. Autistic disorder and schizophrenia: diagnostic overlaps. J Autism Dev Disord 31:19-28.

Larsson HJ, Eaton WW, Madsen KM, Vestergaard M, Olesen AV, Agerbo E, Schendel D, Thorsen P, Mortensen PB. 2005. Risk factors for autism: perinatal factors, parental psychiatric history, and socioeconomic status. Am J Epidemiol 161:916-925.

McClellan J, King MC. 2010. Genomic analysis of mental illness: a changing landscape. JAMA 303:2523-2524.

Merikangas AK, Corvin AP, Gallagher L. 2009. Copy-number variants in neurodevelopmental disorders: promises and challenges. Trends Genet 25:536-544.

Mouridsen SE, Rich B, Isager T. 2008a. Psychiatric disorders in adults diagnosed as children with atypical autism. A case control study. J Neural Transm 115:135-138.

Mouridsen SE, Rich B, Isager T, Nedergaard NJ. 2008b. Psychiatric disorders in individuals diagnosed with infantile autism as children: a case control study. J Psychiatr Pract 14:5-12.

Ozonoff S, Young GS, Carter A, Messinger D, Yirmiya N, Zwaigenbaum L, Bryson S, Carver LJ, Constantino JN, Dobkins K, et al. 2011. Recurrence Risk for Autism Spectrum Disorders: A Baby Siblings Research Consortium Study. Pediatrics.

Pinto D, Pagnamenta AT, Klei L, Anney R, Merico D, Regan R, Conroy J, Magalhaes TR, Correia C, Abrahams BS, et al. 2010. Functional impact of global rare copy number variation in autism spectrum disorders. Nature 466:368-372.

Purcell S, Neale B, Todd-Brown K, Thomas L, Ferreira MA, Bender D, Maller J, Sklar P, de Bakker PI, Daly MJ, et al. 2007. PLINK: a tool set for whole-genome association and population-based linkage analyses. Am J Hum Genet 81:559-575.

Purcell SM, Wray NR, Stone JL, Visscher PM, O'Donovan MC, Sullivan PF, Sklar P. 2009. Common polygenic variation contributes to risk of schizophrenia and bipolar disorder. Nature 460:748-752.

Ruderfer DM, Kirov G, Chambert K, Moran JL, Owen MJ, O'Donovan MC, Sklar P, Purcell SM. 2011. A family-based study of common polygenic variation and risk of schizophrenia. Mol Psychiatry 16:887-888.

Rutter M. 1968. Concepts of autism: a review of research. J Child Psychol Psychiatry 9:1-25.

Rutter M. 1972. Childhood schizophrenia reconsidered. J Autism Child Schizophr 2:315-337.

Sanders SJ, Ercan-Sencicek AG, Hus V, Luo R, Murtha MT, Moreno-De-Luca D, Chu SH, Moreau MP, Gupta AR, Thomson SA, et al. 2011. Multiple Recurrent De Novo CNVs, Including Duplications of the 7q11.23 Williams Syndrome Region, Are Strongly Associated with Autism. Neuron 70:863-885.

Sasson NJ, Pinkham AE, Carpenter KL, Belger A. 2011. The benefit of directly comparing autism and schizophrenia for revealing mechanisms of social cognitive impairment. J Neurodev Disord 3:87-100.

Sherry ST, Ward MH, Kholodov M, Baker J, Phan L, Smigielski EM, Sirotkin K. 2001. dbSNP: the 
NCBI database of genetic variation. Nucleic Acids Res 29:308-311.

Skokauskas N, Gallagher L. 2010. Psychosis, affective disorders and anxiety in autistic spectrum disorder: prevalence and nosological considerations. Psychopathology 43:8-16.

Stahlberg O, Soderstrom H, Rastam M, Gillberg C. 2004. Bipolar disorder, schizophrenia, and other psychotic disorders in adults with childhood onset AD/HD and/or autism spectrum disorders. J Neural Transm 111:891-902.

Stefansson H, Rujescu D, Cichon S, Pietilainen OP, Ingason A, Steinberg S, Fossdal R, Sigurdsson E, Sigmundsson T, Buizer-Voskamp JE, et al. 2008. Large recurrent microdeletions associated with schizophrenia. Nature 455:232-236.

Visscher PM, Goddard ME, Derks EM, Wray NR. 2012. Evidence-based psychiatric genetics, AKA the false dichotomy between common and rare variant hypotheses. Mol Psychiatry 17:474485.

Vorstman JA, Staal WG, van Daalen E, van Engeland H, Hochstenbach PF, Franke L. 2006. Identification of novel autism candidate regions through analysis of reported cytogenetic abnormalities associated with autism. Mol Psychiatry 11:18-28.

Walsh T, McClellan JM, McCarthy SE, Addington AM, Pierce SB, Cooper GM, Nord AS, Kusenda $M$, Malhotra D, Bhandari A, et al. 2008. Rare structural variants disrupt multiple genes in neurodevelopmental pathways in schizophrenia. Science 320:539-543.

Yang J, Benyamin B, McEvoy BP, Gordon S, Henders AK, Nyholt DR, Madden PA, Heath AC, Martin NG, Montgomery GW, et al. 2010. Common SNPs explain a large proportion of the heritability for human height. Nat Genet 42:565-569. 
TABLE I. Variance at Marker $(\mathrm{Vm})$ Explained by P-value Thresholds $(P T)$ of the ISC Schizophrenia Polygene Score in ASD (With and Without HLA Markers)

\begin{tabular}{|c|c|c|c|c|}
\hline Threshold $\left(P_{\mathrm{T}}\right)$ & Cases & $\mathrm{Vm}$ & Sim Vm (SD) & Emp P \\
\hline \multicolumn{5}{|c|}{ All SNPS (N = 33,603) } \\
\hline 0.0001 & 2,736 & 0.0000 & $0.0003(0.0003)$ & 0.93 \\
\hline 0.001 & 2,736 & 0.0005 & $0.0003(0.0003)$ & 0.13 \\
\hline 0.01 & 2,736 & 0.0006 & $0.0002(0.0003)$ & 0.08 \\
\hline 0.05 & 2,736 & 0.0002 & $0.0002(0.0002)$ & 0.46 \\
\hline 0.1 & 2,736 & 0.0001 & $0.0002(0.0002)$ & 0.83 \\
\hline 0.2 & 2,736 & 0.0001 & $0.0003(0.0003)$ & 0.80 \\
\hline 0.3 & 2,736 & 0.0000 & $0.0003(0.0003)$ & 0.86 \\
\hline 0.4 & 2,736 & 0.0000 & $0.0002(0.0003)$ & 0.92 \\
\hline 0.5 & 2,736 & 0.0001 & $0.0003(0.0003)$ & 0.80 \\
\hline \multicolumn{5}{|c|}{ No HLA SNPs $(N=33,457)$} \\
\hline 0.0001 & 2,736 & 0.0000 & $0.0002(0.0002)$ & 0.89 \\
\hline 0.001 & 2,736 & 0.0005 & $0.0003(0.0003)$ & 0.17 \\
\hline 0.01 & 2,736 & 0.0005 & $0.0002(0.0002)$ & 0.06 \\
\hline 0.05 & 2,736 & 0.0002 & $0.0003(0.0003)$ & 0.44 \\
\hline 0.1 & 2,736 & 0.0001 & $0.0003(0.0002)$ & 0.88 \\
\hline 0.2 & 2,736 & 0.0001 & $0.0003(0.0003)$ & 0.90 \\
\hline 0.3 & 2,736 & 0.0000 & $0.0002(0.0003)$ & 0.94 \\
\hline 0.4 & 2,736 & 0.0000 & $0.0002(0.0002)$ & 0.89 \\
\hline 0.5 & 2,736 & 0.0001 & $0.0002(0.0002)$ & 0.90 \\
\hline
\end{tabular}




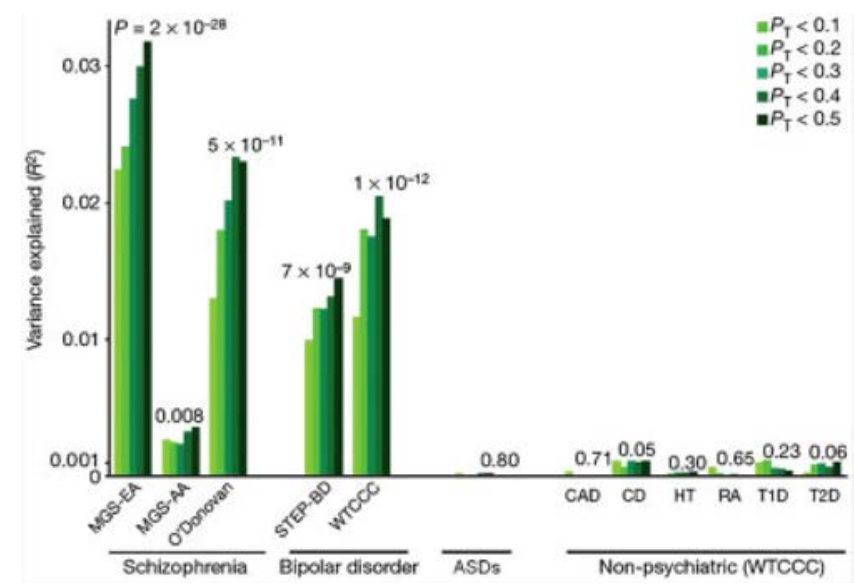

Figure 1: Variance explained by the schizophrenia (ISC)-derived polygenic score in autism spectrum disorders. For the sake of context, the results in the imputed and non-imputed autism (AGP) datasets are inserted in scale into the original figure from the Purcell et al. paper [Purcell et al., 2009]. Note the results from the Purcell et al. paper are based on 74,062 SNPs while the observations of the current study are derived from 33,603 SNPs. However, Purcell and colleagues report that similar results were obtained with 25,215 risk SNPs. Different shades of green represent the five significance thresholds $\left(\mathrm{P}_{\mathrm{T}}\right)$, numbers above the bars indicate empirical $\mathrm{P}$-values with $\mathrm{P}_{\mathrm{T}}=0.5$. $C A D$, coronary artery disease; $C D$, Crohn's disease; $H T$, hypertension; RA, rheumatoid arthritis; T1D, type I diabetes; T2D, type II diabetes.

Proportion of explained variance by common variants with small effect Proportion of explained variance by rare variants with major effect

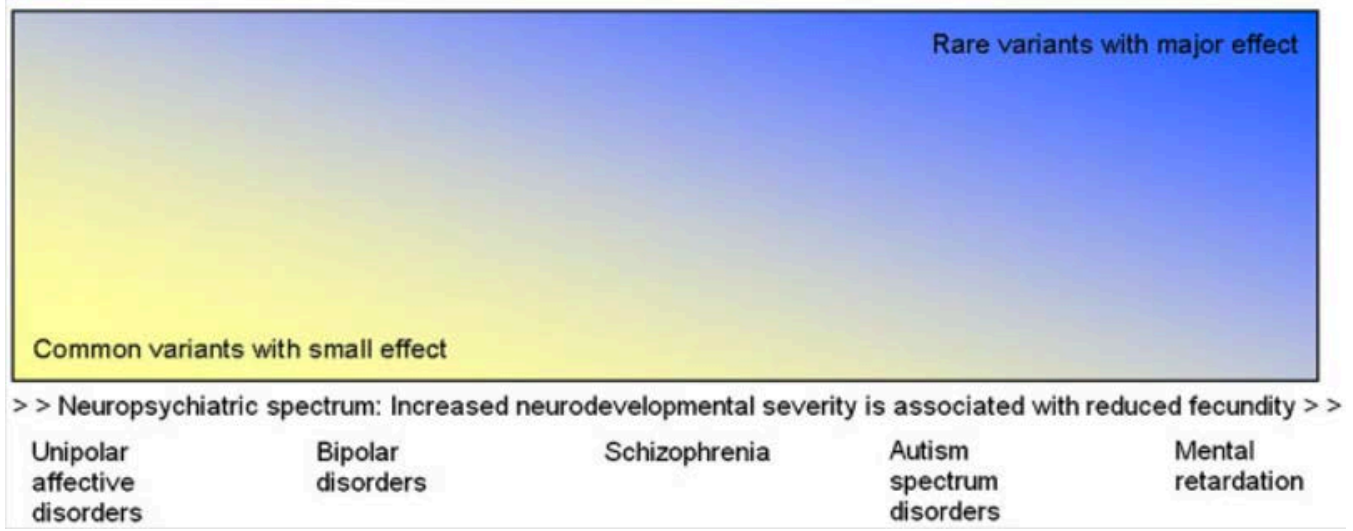

Figure 2: A model for the effect of genetic variants across the neuropsychiatric spectrum. For the purpose of clarity genetic variants are categorized in two classes, common with small effect and rare with major effect. This is likely an oversimplication [Visscher et al., 2012]. In reality variants with variable effect sizes may range from common to very rare, although common variants with large effect size are unlikely. Higher impact variants appear to play a more prominent role in the syndromes at the more severe neurodevelopmental end of the spectrum. The present study suggests that the common variants are likely to be more important at the opposite, less neurodevelopmentally impaired end of the spectrum where the disorders have a relatively smaller impact on fecundity (mood disorders). 